\title{
Weight phobia or overvaluation of shape and weight? A cognitive analysis of the core psychopathology of anorexia nervosa
}

\author{
Riccardo Dalle Grave', Massimiliano Sartirana², Simona Calugi' \\ 'Department of Eating and Weight Disorders, Villa Garda Hospital, Garda (VR), Italy \\ ${ }^{2}$ Associazione Disturbi Alimentari (ADA), Verona, Italy
}

\author{
Key words \\ Anorexia nervosa \\ Weight phobia \\ Overvaluation of shape and weight \\ Cognitive behavioural therapy \\ Exposure
}

\begin{abstract}
The avoidance paradigm has dominated the psychological conceptualization of anorexia nervosa, for many years. According to that theory, "weight phobia" explains most of the behavioural expressions of the disorder, which are adopted to avoid aversive stimuli (i.e., weight gain and getting fat). However, the conceptualization of anorexia nervosa as a simple phobic disorder is difficult to uphold, as it does not explain the sense of triumph, mastery, self-control and superiority that people suffering from this disorder experience when they are able to control their eating and lose weight. Transdiagnostic cognitive behavioural theory, on the other hand, explains the egosyntonic nature of anorexia nervosa, attributing overvaluation of shape, weight and their control as the core psychopathology of the disorder, and considering weight phobia as a secondary expression of this core psychopathology. The evidence-based "enhanced" cognitive behavioural therapy (CBT-E), derived from this transdiagnostic theory, is not designed to primarily address weight phobia, but rather to help patients develop a more articulated and functional scheme of selfevaluation that is less dependent on shape, weight and their control, by involving the patients actively in the implementation of strategies and procedures that make feel them in control during the process of change.
\end{abstract}

Anorexia nervosa is a complex disease whose causes are not yet known, although available data suggest that it seems to derive from the interaction of multiple potential genetic and environmental risk factors (Watson et al., 2019; Zipfel, Giel, Bulik, Hay, \& Schmidt, 2015). In any case, at a certain point, the causal factors of anorexia nervosa converge, bringing the person to develop the belief that they must lose weight.

The pathogenesis of anorexia nervosa has been dominated for many years by a theory based on the avoidance paradigm, according to which the probability of a behaviour increases if it is followed by negative reinforcement (i.e., the removal of the aversive stimulus). If applied to anorexia nervosa, that model apparently explains most of the behavioural expressions of the disorder (e.g., strict dieting, excessive exercising, self-induced vomiting and laxatives and diuretics misuse), which are adopted for the purposed of avoiding specific aversive stimuli, i.e., weight gain and getting fat (Garner \& Bemis, 1982). The model is also compatible with the conceptualization of anorexia

Copyright @ 2019 Riccardo Dalle Grave, Massimiliano Sartirana, Simona Calugi. This is an open-access article distributed under the terms of the Creative Commons Attribution License (CC BY). The use, distribution or reproduction in other forums is permitted, provided the original author(s) and the copyright owner(s) are credited and that the original publication in this journal is cited, in accordance with accepted academic practice. No use, distribution or reproduction is permitted which does not comply with these terms.

Riccardo Dalle Grave, MD (₫) rdalleg@gmail.com

Received: 10 August 2019; Accepted: 19 August 2019; Published online: 27 August 2019. doi:10.32044/ijedo.2019.08 
nervosa, which considers it as an adaptive disorder in which "weight phobia" represents avoidance of the feared circumstances associated with psychosexual maturity (Crisp, 1965). According to this theory, strict dieting and weight loss become a means to avoid the challenges associated with psychosexual development, for which the person with anorexia nervosa feels unprepared.

Although in most cases the source of this phobia (the aversive stimulus) is not expressed by patients, some authors have reported that it is often associated with issues concerning sexuality, high performance standards, separation from family, or family conflicts (Garner \& Bemis, 1982). Then, the theory postulates that the avoidance, once acquired, is maintained by persistence of the behaviours characteristic of eating disorders, which isolate the subject from the possibility of recognizing when the aversive contingencies are no longer operating. Cognitive variables seem to contribute in part to this process, as the avoidance prevents the development of an inhibitory learning of fear by not allowing falsification of the occurrence of the feared consequences (Craske, Treanor, Conway, Zbozinek, \& Vervliet, 2014; Reilly, Anderson, Gorrell, Schaumberg, \& Anderson, 2017). Moreover, as suggested by Beck and Emery (1985), the avoidance behaviour might be perpetuated by "hyperactive" cognitive sets, which operate autonomously; over time, the cognitive anticipation of feared stimuli, for example certain foods or a higher weight reading on the scale, produces anxiety that encourages the patients to adopt strict rules to safeguard themselves against the feared consequences. This terror causes the development of dysfunctional rules that provide a sense of security, such as excluding certain foods and fixing progressively lower weight goals.

However, the conceptualization of anorexia nervosa as a simple phobic disorder is difficult to maintain because the control of shape and weight seen in this disorder is often associated with a sense of triumph, mastery, self-control and superiority in its sufferers (Garner \& Bemis, 1982). It is common to observe this characteristic in the accounts of patients who, especially in the early phases of their weight loss, report being "happy", "exultant", "satisfied", "triumphant", "powerful" and "proud" (Bemis, 1983). Indeed, weight loss is often experienced as a "goal", "an achievement", "a virtue", "a source of positive pleasure" and/or "a pleasure of the senses" (Bemis, 1983). This condition, in which the majority of patients with anorexia nervosa exalt the virtues of the pathological state by actively adopting extreme "ego-syntonic" weight control behaviours (e.g., strict dieting, excessive exercising, self-induced vomiting, and laxative or diuretics misuse), is clearly distinct from simple "weight phobia". Indeed, persons with claustrophobia experience a severe state of anxiety when they are in closed spaces, but they generally do not report a feeling of euphoria or a sense of power when they are outdoors (Garner \& Dalle Grave, 1999). Furthermore, individuals with claustrophobia tend to perceive their anxiety as excessive and uncontrollable, and they use the strategy of avoidance to eliminate this anxiety. Those with anorexia nervosa, on the other hand, often perceive their preoccupation with shape, weight and their control, and the associated negative mood states (e.g., anxiety), as useful means of keeping control over their shape and weight.

The egosyntonic characteristics of anorexia nervosa have led to consider the positive cognitive reinforcement associated with the control of shape and weight as the core psychopathology of the eating disorder, and weight phobia as a secondary expression of this core psychopathology. Indeed, according to the modern transdiagnostic cognitive behavioural theory, a distinctive self-evaluation scheme, the overvaluation of shape, weight, and their control (i.e., judging oneself predominantly or exclusively in terms of shape, weight and their control), is of central importance in maintaining anorexia nervosa, but also other eating disorders (Fairburn, Cooper, \& Shafran, 2003). According to this theory, most other clinical features of the disorder derive directly or indirectly from this specific core psychopathology. For example, extreme weight-control behaviours (e.g., strict dieting, excessive exercising, self-induced vomiting, laxative or diuretics misuse), and associated expressions (body checking and avoidance, feeling fat, preoccupations about shape, weight and eating, and the marginalization of other areas of life), are understandable if a person believes that the control of shape and weight is vital for judging his/her personal value. In addition, dietary restriction and low weight determine the development of numerous symptoms of malnutrition (Calugi, Chignola, El Ghoch, \& Dalle Grave, 2018), which, in turn, contribute to the maintenance of the eating disorder through various distinctive processes. For example, the delayed gastric emptying resulting from malnutrition produces an early sense of fullness even after the ingestion of only modest amounts of food; the social withdrawal secondary to malnutrition, a common feature of these patients, intensifies the use of shape and weight as a means of self-evaluation; and the eating concern secondary to dietary restriction accentuates the adoption of rigid and extreme dietary rules (Dalle Grave, Pasqualoni, \& Marchesini, 2011). Binge eating is the only behavioural expression of eating disorders that does 
not stem directly from their core psychopathology. However, according to transdiagnostic cognitive behavioural theory, this feature, reported by a subgroup of people with anorexia nervosa, derives indirectly from overvaluation of shape and weight and their control; indeed, it is largely maintained by the attempt to adhere to extreme and rigid dietary rules and/or to cope with external events and associated mood changes (Fairburn et al., 2003).

The pursuit of weight loss, therefore, is not merely linked to the attempt to avoid an aversive experience (i.e., having body fat), but is instead a "goal" that provides a system for self-realization and self-evaluation. This also explains why hunger and other malnutrition symptoms are not usually considered as negative by people with anorexia nervosa, but, on the contrary, are often interpreted positively as a sign of self-control and mastery (Dalle Grave, Di Pauli, Sartirana, Calugi, \& Shafran, 2007). Finally, the overvaluation of shape and weight also explains the intensity and duration of the negative reaction reported by people with anorexia nervosa when they experience slight weight gain and/or are unable to control their diet in line with what they have rigidly planned (Fairburn et al., 2003).

Identification of the core psychopathology of anorexia nervosa is important not only in academic speculation; above all, it has relevant implications for the psychological treatment of this disorder. Indeed, 'enhanced' cognitive behavioural therapy (CBT-E) is founded on the transdiagnostic cognitive behavioural conceptualization described above; it ascribes the central role in the maintenance of anorexia nervosa to the overvaluation of shape, weight and their control (rather than weight phobia), and has the main aim of helping patients develop a more articulated and functional scheme of self-evaluation. To reach this goal, the treatment uses specific strategies and procedures (e.g., real-time self-monitoring and the joint construction of a personalized formulation) that have the purpose of educating the patients about the processes that are maintaining their eating disorder and helping them stop identifying themselves with the problem. The patients are then encouraged to make gradual behavioural changes in the context of their personal formulation to evaluate the effects and implications on their way of thinking. Unlike exposure-based programmes (Reilly et al., 2017), the treatment is structured to make the patients feel in control through, for example, planning in advance what food they will eat and gradually introducing foods that they have previously avoided, and aims to create a positive energy balance that will determine a predictable and controlled weekly weight regain. By being able to predict the effects of what they eat on their weight, patients will be more tolerant of the anxiety associated with weight regain and the reintroduction of avoided foods. Furthermore, the treatment actively involves patients in removing the other key expressions of their psychopathology that keep the eating disorder active, helping them develop a more functional and articulated self-evaluation system that is no longer predominantly dependent on shape, weight and their control.

\section{References}

Beck, A. T., \& Emery, G. (1985). Anxiety disorders and phobias: A cognitive perspective. New York: Basic Books.

Bemis, K. M. (1983). A comparison of functional relationships in anorexia nervosa and phobia. In P. L. Darby, P. E. Garfinkel, D. M. Garner, \& D. V. Coscina (Eds.), Anorexia nervosa: Recent developments in research (pp. 403-415). New York: Allan R. Liss.

Calugi, S., Chignola, E., El Ghoch, M., \& Dalle Grave, R. (2018). Starvation symptoms in patients with anorexia nervosa: a longitudinal study. Eating Disorders, 26(6), 523-537. doi:10.1080/10640266.2018.1471921

Craske, M. G., Treanor, M., Conway, C. C., Zbozinek, T., \& Vervliet, B. (2014). Maximizing exposure therapy: an inhibitory learning approach. Behaviour Research and Therapy, 58, 10-23. doi:10.1016/j.brat.2014.04.006

Crisp, A. H. (1965). Clinical and therapeutic aspects of anorexia nervosa--a study of 30 cases. Journal of Psychosomatic Research, 9(1), 67-78.

Dalle Grave, R., Di Pauli, D., Sartirana, M., Calugi, S., \& Shafran, R. (2007). The interpretation of symptoms of starvation/severe dietary restraint in eating disorder patients. Eating and Weight Disorders, 12(3), 108-113.

Dalle Grave, R., Pasqualoni, E., \& Marchesini, G. (2011). Symptoms of starvation in eating disorder patients. In V. R. Preedy (Ed.), Handbook of behavior, food and nutrition (pp. 2259-2269). New York: Springer Science+Business Media.

Fairburn, C. G., Cooper, Z., \& Shafran, R. (2003). Cognitive behaviour therapy for eating disorders: a "transdiagnostic" theory and treatment. Behaviour Research and Therapy, 41(5), 509-528.

Garner,D. M., \& Bemis, K. M. (1982). A cognitive-behavioral approach to anorexia nervosa. Cognitive Therapy and Research, 6(2), 123-150. doi:10.1007/bf01183887

Garner, D. M., \& Dalle Grave, R. (1999). Terapia cognitivo comportamentale dei disturbi dell'alimentazione. Verona: Positive Press. 
Reilly, E. E., Anderson, L. M., Gorrell, S., Schaumberg, K., \& Anderson, D. A. (2017). Expanding exposure-based interventions for eating disorders. International Journal of Eating Disorders, 50(10), 1137-1141. doi:10.1002/ eat. 22761

Watson, H. J., Yilmaz, Z., Thornton, L. M., Hübel, C., Coleman, J. R. I., Gaspar, H. A., . . Bulik, C. M. (2019). Genome-wide association study identifies eight risk loci and implicates metabo-psychiatric origins for anorexia nervosa. Nature Genetics. doi:10.1038/ s41588-019-0439-2

Zipfel, S., Giel, K. E., Bulik, C. M., Hay, P., \& Schmidt, U. (2015). Anorexia nervosa: aetiology, assessment, and treatment. Lancet Psychiatry, 2(12), 1099-1111. doi:10.1016/s2215-0366(15)00356-9 